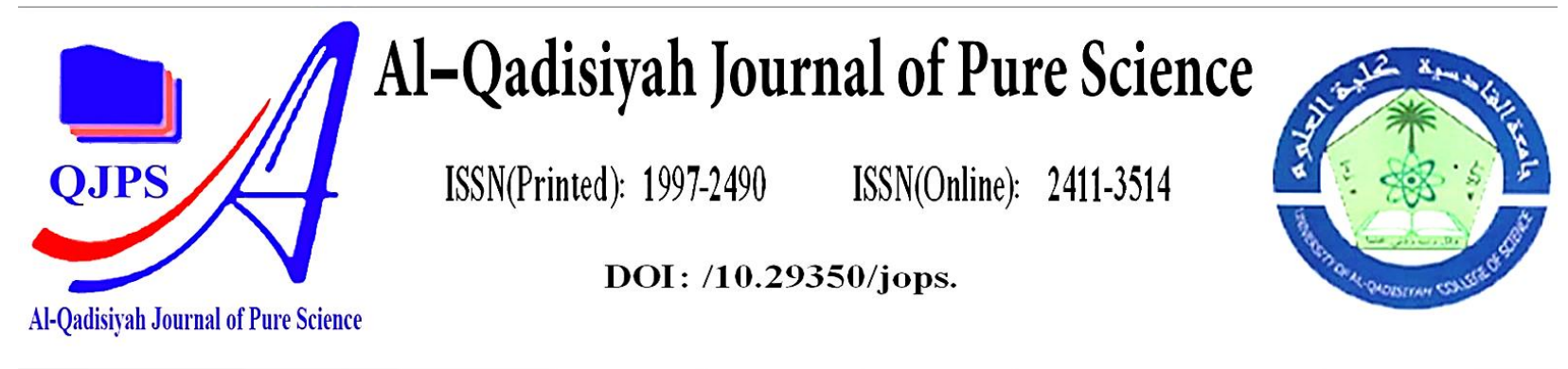

\title{
A New Class of Harmonic Univalent Functions of the Salagean Type
}

\begin{tabular}{|c|c|}
\hline Authors Names & ABSTRACT \\
\hline $\begin{array}{l}\text { a.Mustafa I. Hameed } \\
\text { b.Buthyna Najad Shihab }\end{array}$ & $\begin{array}{l}\text { A new family of Salagean type harmonic univalent functions is described and } \\
\text { investigated. For the functions in this class, we derive coefficient inequalities, } \\
\text { extreme points, and distortion limits. }\end{array}$ \\
\hline Article History & \\
\hline Receivedon: 18/6/2021 & \\
\hline Revisedon:30/7/2021 & \\
\hline Accepted on:15/8/2021 & \\
\hline Keywords: & \\
\hline Harmonic Univalent Functions, & \\
\hline Salagean Derivative, Extreme & \\
\hline Points, Distortion & \\
\hline DOI: & \\
\hline $\begin{array}{l}\text { https://doi.org/10.29350/ } \\
\text { jops.2021.26. } 4.1387\end{array}$ & \\
\hline & \\
\hline & \\
\hline
\end{tabular}




\section{INTRODUCTION}

If both $t$ and $l$ are real harmonic in $E$, a continuous complex-valued function $w=t+i l$ defined in a simply connected complex domain $E$ is said to be harmonic in $E$. We may write $w=k+\bar{f}$ in any simply connected domain, where $k$ and $f$ are analytic in $E .\left|k^{\prime}(u)\right|>\left|f^{\prime}(u)\right|, u \in E$ is an essential and sufficient condition for $w$ to be locally univalent and sense preserving in $E$.

$S_{T}$ denotes the class of harmonic univalent and sense-preserving functions $w=k+\bar{f}$ in the unit disk $U=$ $\{u:|u|<1\}$ for which $w(0)=w_{u}(0) 1=0$. Then, for $w=k+\bar{f} \in S_{T}$, the analytic functions $k$ and $f$ can be expressed as

$$
k(u)=u+\sum_{m=2}^{\infty} d_{m} u^{m}, \quad f(u)=\sum_{m=1}^{\infty} e_{m} u^{m}, \quad\left(\left|e_{1}\right|<1, u \in U\right)
$$

Clunie and Sheil Small [8] studied the class $S_{T}$ and its geometric subclasses in 1984 and came up with some coefficient bounds. Since then, several papers on $S_{T}$ and its subclasses have been written.

Salagean [17] introduced the differential operator $E^{V}$. More details can be seen in [2], [4], [5], [6], [7] and [20]. Jahangiri et al. [13] defined the modified Salagean operator of $w$ as for $w=k+\bar{f}$ given by (1).

where

$$
E^{v} w(u)=E^{v} k(u)+(-1)^{v} \overline{E^{v} f(u)}
$$

$$
E^{v} k(u)=u+\sum_{m=2}^{\infty} m^{v} d_{m} u^{m}, \quad E^{v} f(u)=\sum_{m=1}^{\infty} m^{v} e_{m} u^{m} .
$$

Let $S_{T}(v, r, \tau, \gamma)$ denote the class of univalent harmonic functions of the form (1) that satisfy the condition for fixed positive integers $v, r, 0 \leq \tau<1$ and $\gamma \geq 0$

$$
\mathcal{R} e\left\{\frac{E^{v} w(u)}{E^{r} w(u)}\right\}>\left|\gamma \frac{E^{v} w(u)}{E^{r} w(u)}-\tau\right|,
$$

where $E^{v} w(u)$ is defined by (2).

The subset $\bar{S}_{T}(v, r, \tau, \gamma)$ is made up of harmonic functions. In $\bar{S}_{T}(v, r, \tau, \gamma), w_{v}=k+\bar{f}_{v}$, where $k$ and $f_{v}$ are of the form

$$
k(u)=u-\sum_{m=2}^{\infty} d_{m} u^{m}, \quad f_{v}(u)=(-1)^{v-1} \sum_{m=1}^{\infty} e_{m} u^{m} ; d_{m}, e_{m} \geq 0 .
$$

A number of well-known $S_{T}$ subclasses are included in the class $\bar{S}_{T}(v, r, \tau, \gamma) . \bar{S}_{T}(1,0, \tau, 0) \equiv \mathrm{F}(\tau)$ is a class of sense-preserving, harmonic univalent functions $w$ that are starlike of order $\tau$ in $U, \bar{S}_{T}(2,1, \tau, 0)$ is a class of sensepreserving, harmonic univalent functions $w$ that are convex of order $\tau$ in $U$, and $\bar{S}_{T}(r+1, r, \tau, 0) \equiv \bar{T}(r, \tau)$ is a class of Salagean type harmonic univalent functions. Avc1 and Zlotkiewicz [3] demonstrated that if the harmonic function $w$ of the form (1) has $e_{1}=0$,

$$
\sum_{m=2}^{\infty} m\left(\left|d_{m}\right|+\left|e_{m}\right|\right) \leq 1
$$

then $w \in T S(0)$ and if

$$
\sum_{m=2}^{\infty} m^{2}\left(\left|d_{m}\right|+\left|e_{m}\right|\right) \leq 1
$$

then $w \in T k(0)$. Silverman [18] demonstrated that if $w=k+\bar{f}$ has negative coefficients, the above coefficient condition is also needed. Later, Silverman and Silvia [19] strengthened [1], [10], [14], [15] and [16] are results for the case $e_{1}$ that isn't necessarily zero. 
Jahangiri [9], [11] and [12] demonstrated that for harmonic functions $w$ of the form (4) with $v=1, w \in \mathrm{F}(\tau)$ if and only if

and $w \in \bar{S}_{T}(2,1, \tau, 0)$ if and only if

$$
\sum_{m=2}^{\infty}(m-\alpha)\left|d_{m}\right|+\sum_{m=1}^{\infty}(m+\alpha)\left|e_{m}\right| \leq 1-\alpha
$$

$$
\sum_{m=2}^{\infty} m(m-\alpha)\left|d_{m}\right|+\sum_{m=1}^{\infty} m(m+\alpha)\left|e_{m}\right| \leq 1-\alpha
$$

The above results are applied in this note to the families $S_{T}(v, r, \tau, \gamma)$ and $\bar{S}_{T}(v, r, \tau, \gamma)$. For $\bar{S}_{T}(v, r, \tau, \gamma)$, we also get extreme points, distortion bounds, convolution conditions, and convex combinations.

\section{MAIN RESULTS}

For harmonic functions in $S_{T}(v, r, \tau, \gamma)$, we introduce an adequate coefficient bound in our first theorem.

Theorem 2.1. Let $w=k+\bar{f}$ be so that $k$ and $f$ are given by (1). Furthermore, let

$$
\sum_{m=1}^{\infty}\left(\frac{(\tau-1) m^{r}+(1-\gamma) m^{v}}{(1+\tau-\gamma)}\left|d_{m}\right|+\frac{(\tau-1) m^{r}+(-1)^{v-r}(1-\gamma) m^{v}}{(1+\tau-\gamma)}\left|e_{m}\right|\right) \leq 2
$$

where $d_{1}=1, v \in \mathbb{N}, r \in \mathbb{N}_{0}, v>r$ and $0 \leq \tau<1, \beta \geq 0$; then $w$ is sense-preserving, harmonic univalentin $U$ and $w \in S_{T}(v, r, \tau, \gamma)$.

Proof. According to (2) and (4) we only need to show that

$$
\begin{gathered}
\left|\frac{(1+2 \tau) E^{r} w(u)+E^{v} w(u)-(1+\tau) E^{r} w(u)-\gamma E^{v} w(u)}{E^{r} w(u)}\right|>0 \\
=\left|\frac{\begin{array}{c}
(1+2 \tau)\left[E^{r} k(u)+(-1)^{r} \overline{E^{r} f(u)}\right]+\left[E^{v} k(u)+(-1)^{v} \overline{E^{v} f(u)}\right]- \\
(1+\tau)\left[E^{r} k(u)+(-1)^{r} \overline{E^{r} f(u)}\right]-\gamma\left[E^{v} k(u)+(-1)^{v} \overline{E^{v} f(u)}\right]
\end{array} \mid}{E^{r} w(u)}\right|
\end{gathered}
$$

$$
\begin{aligned}
& =\left|\begin{array}{c}
(1+\tau-\gamma) u+\sum_{m=2}^{\infty}\left(\tau m^{r}+(1-\gamma) m^{v}\right) d_{m} u^{m} \\
+(-1)^{r} \sum_{m=1}^{\infty}\left(\tau m^{r}+(-1)^{v-r}(1-\gamma) m^{v}\right) \overline{e_{m}} u^{m} \\
u+\sum_{m=2}^{\infty} m^{r} d_{m} u^{m}+(-1)^{r} \sum_{m=1}^{\infty} m^{r} \overline{e_{m} u^{m}}
\end{array}\right| \\
& \geq\left(\begin{array}{c}
(1+\tau-\gamma)|u|+\sum_{m=2}^{\infty}\left(\tau m^{r}+(1-\gamma) m^{v}\right)\left|d_{m}\right|\left|u^{m}\right| \\
+\sum_{m=1}^{\infty}\left(\tau m^{r}+(-1)^{v-r}(1-\gamma) m^{v}\right)\left|e_{m}\right|\left|u^{m}\right| \\
|u|+\sum_{m=2}^{\infty} m^{r}\left|d_{m}\right|\left|u^{m}\right|+(-1)^{r} \sum_{m=1}^{\infty} m^{r}\left|e_{m}\right|\left|u^{m}\right|
\end{array}\right) \\
& \left\{\begin{array}{l}
(1+\tau-\gamma)|u|+\sum_{m=2}^{\infty}\left(\tau m^{r}+(1-\gamma) m^{v}\right)\left|d_{m}\right|\left|u^{m}\right|+\sum_{m=1}^{\infty}\left(\tau m^{r}-(1-\gamma) m^{v}\right)\left|e_{m}\right|\left|u^{m}\right| \text { if } v-r \text { is odd } \\
(1+\tau-\gamma)|u|+\sum_{m=2}^{\infty}\left(\tau m^{r}+(1-\gamma) m^{v}\right)\left|d_{m}\right|\left|u^{m}\right|+\sum_{m=1}^{\infty}\left(\tau m^{r}+(1-\gamma) m^{v}\right)\left|e_{m}\right|\left|u^{m}\right| \text { if } v-r \text { is even }
\end{array}\right\}
\end{aligned}
$$




$$
\begin{gathered}
=(1+\tau-\gamma)|u|\left\{1+\sum_{m=2}^{\infty} \frac{\tau m^{r}+(1-\gamma) m^{v}}{(1+\tau-\gamma)}\left|d_{m}\right||u|^{m-1}+\sum_{m=1}^{\infty} \frac{\tau m^{r}+(-1)^{v-r}(1-\gamma) m^{v}}{(1+\tau-\gamma)}\left|e_{m}\right||u|^{m-1}\right\} \\
>(1+\tau-\gamma)\left\{1+\sum_{m=2}^{\infty} \frac{\tau m^{r}+(1-\gamma) m^{v}}{(1+\tau-\gamma)}\left|d_{m}\right|+\sum_{m=1}^{\infty} \frac{\tau m^{r}+(-1)^{v-r}(1-\gamma) m^{v}}{(1+\tau-\gamma)}\left|e_{m}\right|\right\} .
\end{gathered}
$$

Since (6) makes this last expression non-negative, the proof is complete. The harmonic univalent functions

$$
w(u)=u+\sum_{m=2}^{\infty} \frac{1+\tau-\gamma}{\tau m^{r}+(1-\gamma) m^{v}} x_{m} u^{m}+\sum_{m=1}^{\infty} \frac{1+\tau-\gamma}{\tau m^{r}+(-1)^{v-r}(1-\gamma) m^{v}} \overline{y_{m}} u^{m},
$$

where $v \in \mathbb{N}, r \in \mathbb{N}_{0}, v>r$ and $\sum_{m=2}^{\infty}\left|x_{m}\right|+\sum_{m=1}^{\infty}\left|y_{m}\right|=1$, demonstrate the sharpness of the coefficient bound given by (6). $S_{T}(v, r, \tau, \gamma)$ contains functions of the form (8).

$$
\sum_{m=1}^{\infty}\left(\frac{\tau m^{r}+(1-\gamma) m^{v}}{(1+\tau-\gamma)}\left|d_{m}\right|+\frac{\tau m^{r}+(-1)^{v-r}(1-\gamma) m^{v}}{(1+\tau-\gamma)}\left|e_{m}\right|\right)=1+\sum_{m=2}^{\infty}\left|x_{m}\right|+\sum_{m=1}^{\infty}\left|y_{m}\right|=2 .
$$

The condition (6) is also required for functions $w_{v}=k+\bar{f}_{v}$ where $k$ and $f_{v}$ are of the form (5) shown in the following theorem.

Theorem 2.2. Let $w_{v}=k+\bar{f}_{v}$ be given by (5). Then $w_{v} \in \bar{S}_{T}(v, r, \tau, \gamma)$ if and only if

$$
\sum_{m=1}^{\infty}\left[\left(\tau m^{r}+(1-\gamma) m^{v}\right) d_{m}+\left(\tau m^{r}+(-1)^{v-r}(1-\gamma) m^{v}\right) e_{m}\right] \leq 2(1+\tau-\gamma),
$$

where $d_{1}=1,0 \leq \tau<1, \gamma \geq 0 v \in \mathbb{N}, r \in \mathbb{N}_{0}, v>r$.

Proof. We just need to prove the only if part of the theorem since $\bar{S}_{T}(v, r, \tau, \gamma) \subset S_{T}(v, r, \tau, \gamma)$. In order to do this, for functions $w_{v}$ of the form (5), we are mindful of the situation

$\mathcal{R} e\left\{\frac{E^{v} w(u)}{E^{r} w(u)}\right\}>\left|\gamma \frac{E^{v} w(u)}{E^{r} w(u)}-\tau\right|$ or equivalent to

$$
\mathcal{R} e\left(\begin{array}{c}
(1+\tau-\gamma) u-\sum_{m=2}^{\infty}\left(\tau m^{r}+(1-\gamma) m^{v}\right) d_{m} u^{m} \\
+(-1)^{v+r-1} \sum_{m=1}^{\infty}\left(\tau m^{r}+(-1)^{v-r}(1-\gamma) m^{v}\right) e_{m} \bar{u}^{m} \\
u-\sum_{m=2}^{\infty} m^{r} d_{m} u^{m}+(-1)^{v+r-1} \sum_{m=1}^{\infty} m^{r} e_{m} \bar{u}^{m}
\end{array}\right) \geq 0 .
$$

For all values of $u$ in $U$, the necessary condition (10) must hold. We must have $0 \leq u=z<1$, when choosing the values of $u$ on the positive real axis

$$
\left(\begin{array}{c}
(1+\tau-\gamma)-\sum_{m=2}^{\infty}\left(\tau m^{r}+(1-\gamma) m^{v}\right) d_{m} z^{m-1} \\
\frac{-(-1)^{v-r} \sum_{m=1}^{\infty}\left(\tau m^{r}+(-1)^{v-r}(1-\gamma) m^{v}\right) e_{m} z^{m-1}}{u-\sum_{m=2}^{\infty} m^{r} d_{m} z^{m}-(-1)^{v-r} \sum_{m=1}^{\infty} m^{r} e_{m} z^{m-1}}
\end{array}\right) \geq 0 .
$$

If condition (9) is not satisfied, the numerator in (11) is negative for $z$ near enough to 1 . As a result, for $u_{0}=z_{0}$ in $(0,1)$, the quotient in $(11)$ is negative. The proof is complete since this contradicts the necessary condition for $w_{v} \in \bar{S}_{T}(v, r, \tau, \gamma)$. 
The extreme points of closed convex hulls of $\bar{S}_{T}(v, r, \tau, \gamma)$ denoted by clco $\bar{S}_{T}(v, r, \tau, \gamma)$ are then determined

Theorem 2.3. Let $w_{v}=k+\bar{f}_{v}$ be given by (5). Then $w_{v} \in \bar{S}_{T}(v, r, \tau, \gamma)$ if and only if

where

$$
w_{v}(u)=\sum_{m=1}^{\infty}\left(x_{m} k_{m}(u)+y_{m} f_{v_{m}}(u)\right)
$$

and

$$
k_{1}(u)=u, \quad k_{m}(u)=u-\frac{1+\tau-\gamma}{\tau m^{r}+(1-\gamma) m^{v}} u^{m}, \quad(m=2,3, \ldots)
$$

$$
f_{v_{m}}(u)=u+(-1)^{v-1} \frac{1+\tau-\gamma}{\tau m^{r}+(-1)^{v-r}(1-\gamma) m^{v}} \bar{u}^{m}, \quad(m=1,2, \ldots),
$$

$x_{m} \geq 0, y_{m} \geq 0, x_{1}=1-\sum_{m=2}^{\infty}\left(x_{m}+y_{m}\right) \geq 0$. In particular, the extreme points of $\bar{S}_{T}(v, r, \tau, \gamma)$ are $\left\{k_{m}\right\}$ and $\left\{f_{v_{m}}\right\}$.

Proof. Suppose

$$
\begin{gathered}
w_{v}(u)=\sum_{m=1}^{\infty}\left(x_{m} k_{m}(u)+y_{m} f_{v_{m}}(u)\right) \\
=\sum_{m=1}^{\infty}\left(x_{m}+y_{m}\right) u-\sum_{m=2}^{\infty} \frac{1+\tau-\gamma}{\tau m^{r}+(1-\gamma) m^{v}} x_{m} u^{m}+(-1)^{v-1} \sum_{m=1}^{\infty} \frac{1+\tau-\gamma}{\tau m^{r}+(-1)^{v-r}(1-\gamma) m^{v}} y_{m} \bar{u}^{m} .
\end{gathered}
$$

Then

$$
\begin{gathered}
\sum_{m=2}^{\infty} \frac{\tau m^{r}+(1-\gamma) m^{v}}{1+\tau-\gamma}\left(\frac{1+\tau-\gamma}{\tau m^{r}+(1-\gamma) m^{v}} x_{m}\right) \\
+\sum_{m=1}^{\infty} \frac{\tau m^{r}+(-1)^{v-r}(1-\gamma) m^{v}}{1+\tau-\gamma}\left(\frac{1+\tau-\gamma}{\tau m^{r}+(-1)^{v-r}(1-\gamma) m^{v}} y_{m}\right) \\
=\sum_{m=2}^{\infty} x_{m}+\sum_{m=1}^{\infty} y_{m}=1-x_{1} \leq 1
\end{gathered}
$$

and so $w_{v} \in \operatorname{clco} \bar{S}_{T}(v, r, \tau, \gamma)$.

Conversely, if $w_{v} \in \operatorname{clco} \bar{S}_{T}(v, r, \tau, \gamma)$, then $d_{m} \leq \frac{1+\tau-\gamma}{\tau m^{r}+(1-\gamma) m^{v}}$ and $e_{m} \leq \frac{1+\tau-\gamma}{\tau m^{r}+(-1)^{v-r}(1-\gamma) m^{v}}$. Set

$$
x_{m}=\frac{\tau m^{r}+(1-\gamma) m^{v}}{1+\tau-\gamma} d_{m},(m=2,3, \ldots), \text { and } y_{m}=\frac{\tau m^{r}+(-1)^{v-r}(1-\gamma) m^{v}}{1+\tau-\gamma} e_{m},(m=1,2, \ldots) \text {. }
$$

Then note that by Theorem 2.2, $0 \leq x_{m} \leq 1,(m=2,3, \ldots)$ and $0 \leq y_{m} \leq 1,(m=1,2, \ldots)$. We define

$$
x_{1}=1-\sum_{k=2}^{\infty} x_{k}-\sum_{k=1}^{\infty} y_{k}
$$

and note that, by Theorem 2.2, $x_{1} \geq 0$. Consequently, we obtain

$$
w_{v}(u)=\sum_{m=1}^{\infty}\left(x_{m} k_{m}(u)+y_{m} g f_{v_{m}}(u)\right) \text {. }
$$

The distortion limits for functions in $\bar{S}_{T}(v, r, \tau, \gamma)$ are given by the following theorem, which yields a covering result for this class.

Theorem 2.4. Let $w_{v} \in \bar{S}_{T}(v, r, \tau, \gamma)$. Then for $|u|=z<1$, we have

$$
\left|w_{v}(u)\right| \leq\left(1+e_{1}\right) z+\frac{1}{2^{n}}\left(\frac{\alpha-\beta}{(1-\beta) 2^{m-n}+(\alpha-1)}-\frac{(\alpha-1)+(-1)^{m-n}(1-\beta)}{(1-\beta) 2^{m-n}+(\alpha-1)} b_{1}\right) r^{2},
$$


and

$$
\left|w_{v}(u)\right| \geq\left(1+e_{1}\right) z-\frac{1}{2^{n}}\left(\frac{\alpha-\beta}{(1-\beta) 2^{m-n}+(\alpha-1)}-\frac{(\alpha-1)+(-1)^{m-n}(1-\beta)}{(1-\beta) 2^{m-n}+(\alpha-1)} b_{1}\right) r^{2}
$$

Proof. Only the right hand inequality is proven. The left-hand inequality's proof is identical and will be omitted. Let $w_{v} \in \bar{S}_{T}(v, r, \tau, \gamma)$. be the function. Taking $w_{v}$ is absolute meaning, we get

$$
\begin{gathered}
\left|w_{v}(u)\right|=\left|u-\sum_{m=2}^{\infty} d_{m} u^{m}+(-1)^{v-1} \sum_{m=1}^{\infty} e_{m} u^{m}\right| \leq z+\sum_{m=2}^{\infty} d_{m} z^{m}+(-1)^{v-1} \sum_{m=1}^{\infty} e_{m} z^{m} \\
\leq\left(1+e_{1}\right) z+\sum_{m=2}^{\infty}\left(d_{m}+e_{m}\right) z^{m} \leq\left(1+e_{1}\right) z+\sum_{m=2}^{\infty}\left(d_{m}+e_{m}\right) z^{2} \\
=\left(1+e_{1}\right) z+\frac{\tau-\gamma}{2^{r}\left[(1-\gamma) 2^{v-r}+(\tau-1)\right]} \sum_{m=2}^{\infty} \frac{2^{r}\left[(1-\gamma) 2^{v-r}+(\tau-1)\right]}{\tau-\gamma}\left(d_{m}+e_{m}\right) z^{2} \\
\leq\left(\left(1+e_{1}\right) z+\frac{(\tau-\gamma) z^{2}}{2^{r}\left[(1-\gamma) 2^{v-r}+(\tau-1)\right]}\right) \times \\
\sum_{m=2}^{\infty}\left(\frac{(\tau-1) m^{r}+(1-\gamma) m^{v}}{\tau-\gamma} d_{m}+\frac{(\tau-1) m^{r}+(-1)^{v-r}(1-\gamma) m^{v}}{\tau-\gamma} e_{m}\right) \\
\leq\left(1+e_{1}\right) z+\frac{1}{2^{r}}\left(\frac{\tau-\gamma}{(1-\gamma) 2^{v-r}+(\tau-1)}-\frac{(\tau-1) m^{r}+(-1)^{v-r}(1-\gamma)}{(1-\gamma) 2^{v-r}+(\tau-1)} e_{1}\right) z^{2} .
\end{gathered}
$$

The left hand inequality in Theorem 2.4 leads to the following covering result.

Corollary 2.5. Let $w_{v} \in \bar{S}_{T}(v, r, \tau, \gamma)$, then for $|u|=z<1$, we have

$$
\begin{aligned}
\left\{h:|h|<\frac{\left(\gamma 2^{-v}+1-\gamma\right) 2^{v}+\left(\tau-1-\tau 2^{-r}\right) 2^{r}}{(1-\gamma) 2^{v}+(\tau-1) 2^{r}}\right. & \\
\left.-\frac{\left.\left(\tau-1-(\tau-1) 2^{-r}\right) 2^{r}-((-1))^{v-r}(1-\gamma) 2^{-v}-1+\gamma\right) 2^{v}+}{(1-\gamma) 2^{v}+(\tau-1) 2^{r}}\right\} & \subset w_{v}(U) .
\end{aligned}
$$

Remark 2.6. If we take $v=r+1, \gamma=0$, then the above covering result given in [3]. Furthermore, the results of this paper, for $\gamma=0$ coincide with the results in [4].

\section{REFERENCES}

[1] O.P. Ahuja, A. Çetinkaya, V. Ravichandran, Harmonic univalent functions defined by post quantum calculus operators, Acta Univ. Sapientiae, Mathematica 11(1), (2019), 5-17.

[2] S. Altınkaya, S. Çakmak, S. Yalçın, On a new class of Salagean-type harmonic univalent functions associated with subordination, Honam Math J 40(3), (2018), 433-446.

[3] Y. Avc1, E. Zlotkiewicz, On harmonic univalent mappings, Ann. Univ. Mariae Curie Sklodowska Sect. A 44 (1990) $1-7$. 
[5] H. Bayram, A Subclass of Harmonic Univalent Functions Defined by Salagean Integro Differential Operator, Al-Qadisiyah Journal Of Pure Science 25.3 (2020), 62-70.

[6] H. Bayram, S. Yalçın, A SUBCLASS OF HARMONIC UNIVALENT FUNCTIONS DEFINED BY SUBORDINATION, Acta Universitatis Apulensis, No. 60/2019, pp. 91-103.

[7] H. Bayram, S. Yalçin, On a new subclass of harmonic univalent functions, Malaysian Journal of Mathematical Sciences 14.1 (2020), 63-75.

[8] J. Clunie, T. Sheil-Small, Harmonic univalent functions, Ann. Acad. Sci. Fenn. Ser. A I Math. 9 (1984) 3-25.

[9] J. Dziok, JM. Jahangiri, H. Silverman, Harmonic functions with varying coefficients. J Inequal Appl 16,139, 2016.

[10] M.I. Hameed, C. Ozel, A. Al-Fayadh and A.R.S. Juma, Study of certain subclasses of analytic functions involving convolution operator, AIP Conference Proceedings, Vol. 2096. No. 1. AIP Publishing LLC, 2019.

[11] M. Hameed and I. Ibrahim, Some Applications on Subclasses of Analytic Functions Involving Linear Operator, 2019 International Conference on Computing and Information Science and Technology and Their Applications (ICCISTA). IEEE, 2019.

[12] J.M. Jahangiri, Harmonic functions starlike in the unit disk, J. Math. Anal. Appl. 235 (1999) 470-477.

[13] J.M. Jahangiri, G. Murugusundaramoorthy, K. Vijaya, Salagean-type harmonic univalent functions, South. J. Pure Appl.Math. 2 (2002) 77-82.

[14] S. Jameel Al-Dulaimi and M. I. Hameed, Applications Of Generalized Hypergeometric Analysis Function Of Second Order Differential Subordination, Turkish Journal of Computer and Mathematics Education Vol.12 No. 9 (2021), 3485-3490.

[15] A.R.S. Juma, R.A. Hameed and M.I. Hameed, Certain subclass of univalent functions involving fractional qcalculus operator, Journal of Advance in Mathematics 13.4, 2017.

[16] A.R.S. Juma, R.A. Hameed and M.I. Hameed, SOME RESULTS OF SECOND ORDER DIFFERENTIAL SUBORDINATION INVOLVING GENERALIZED LINEAR OPERATOR., Acta Universitatis Apulensis No. (53), pp. 19-39, 2018.

[17] G.S. Salagean, Subclass of univalent functions, Complex Analysis-Fifth Romanian Finish Seminar, Bucharest, 1, 1983,pp. 362-372.

[18] H. Silverman, Harmonic univalent function with negative coefficients, J. Math. Anal. Appl. 220 (1998) 283289.

[19] H. Silverman, E.M. Silvia, Subclasses of harmonic univalent functions, New Zealand J. Math. 28 (1999) 275284.

[20] S. Yalcin, A new class of Salagean-type harmonic univalent functions, Applied Mathematics Letters, Vol.18, 2(2005), 191-198. 